\section{THE INNOVATIVE NEW AUTOCLAVE}

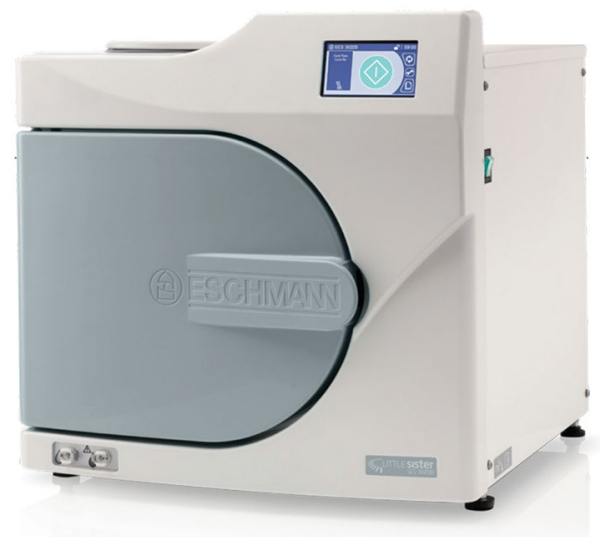

Eschmann has recently launched its new Little Sister SES 3020B autoclave - a versatile option for professionals who want to keep at the very cutting-edge of infection control.

An innovatively designed system that can comfortably fit on a standard work surface, the new autoclave features a large, 23-litre capacity chamber and can process up to $6 \mathrm{~kg}$ of instruments. Plus, due to its dedicated long instrument tray, it can process instruments up to $370 \mathrm{~mm}$ in length.

The system boasts a number of features making it a must-have for the modern professional, including the ability to switch between 'N' type and 'B' type cycles to accommodate every type of load. Furthermore, inbuilt multiple processors guarantee the sterility of each cycle, keeping compliance at the forefront of your infection control process.

To find out more about the state-ofthe-art Little Sister SES 3020B, contact the team at Eschmann.

For more information on the highly effective and affordable range of decontamination equipment and products from Eschmann, visit www. eschmann.co.uk or call 01903875787.

\title{
A COMPREHENSIVE RECYCLING SOLUTION FOR DENTAL PRACTICES
}

TerraCycle's Oral Care Waste and Packaging Zero Waste Box allows dental practices to offer staff and patients a recycling solution for their used toothbrushes, toothpaste tubes and much more.

Consumers are increasingly looking to make more sustainable choices as they go about their daily lives. When it comes to oral care, a patient's health is the top priority, and unfortunately plastic waste is unavoidable. Thankfully, TerraCycle's team of engineers, polymer scientists and recycling experts have over 20 years of experience in keeping otherwise unrecyclable materials in use and out of landfills and incineration, and have come up with a solution specifically for used oral care products.

The Oral Care Waste and Packaging Zero Waste Box is the first comprehensive recycling solution for hard-to-recycle oral care products and packaging, developed by the awardwinning waste experts at TerraCycle.

Whether you're looking to recycle toothpaste tubes and caps, plastic toothbrushes, electric toothbrush heads which don't contain an RFID microchip or interdental brushes and floss containers, the Zero Waste Box system is an all-inclusive recycling solution for all of those dental items that are typically considered 'non-recyclable' through traditional council recycling systems.

The Zero Waste Box system is convenient and easy to use, making it the perfect option for dental practices looking to offset their impacts and reduce their environmental footprint. Aside from the obvious environmental benefits, an Oral Care Waste and Packaging Zero Waste Box also offers advantages such as bolstering a practice's reputation within the local community, singling them out as a positive changemaker for a more sustainable planet, generating potential positive word of mouth, increasing engagement with their existing patients, and

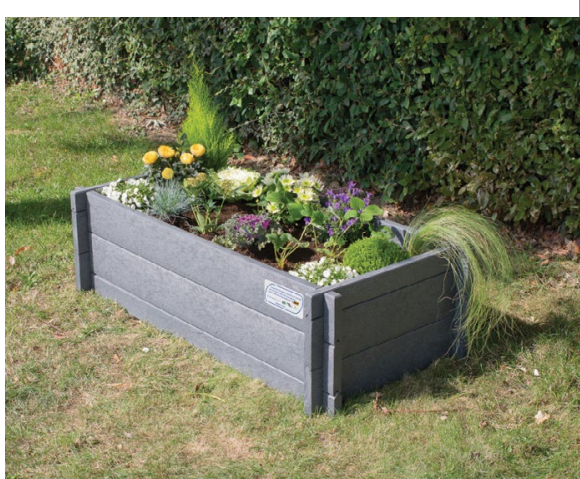

even gaining new patients.

Zero Waste Boxes come in one of three sizes, small, medium and large, with prices ranging from $£ 134.21$ for a small box, which will hold approximately 250 waste items, to $£ 263.71$ for a large box which will hold around 1,250 items. The price of each box includes the cost of TerraCycle sending it to the customer, the prepaid shipping label which the customer then uses to send their full box back to TerraCycle, and the cost of processing the waste.

In order to recycle this waste, TerraCycle partners with a number of third-party processors who turn the waste into a reusable raw material. The waste sent for recycling is shredded, washed and turned into an agglomerate or pellets. These pellets can then be melted down and moulded by manufacturers into new products such as outdoor furniture, planters (pictured) and even playgrounds for schools.

If you are interested in introducing a Zero Waste Box to your practice, giving a boost to your corporate social responsibility and demonstrating your action to protect the environment, or if you're looking for ways to increase footfall and positive feedback from patients, then find out more at https:// www.terracycle.com/en-GB. https:// zerowasteboxes.terracycle.co.uk/

\section{SO MANY REASONS TO GET INVOLVED}

The British Society of Dental Hygiene and Therapy (BSDHT) is committed to helping professionals have their voices heard and expand in their careers. This is why they offer their members a number of exceptional perks that help them to explore new avenues within the profession.

For example, members will receive a subscription to Dental Health - the BSDHT's respected clinical journal which is a trusted source for all of the latest developments and research in the industry.

Members will also receive discounted rates to flagship events such as the Oral Health Conference - the best event for dental hygienists, dental therapists and students of the profession where you can network, learn and more. So, why not contact the BSDHT today and find out how we can support your professional journey?

One of the core values of the BSDHT is inspiring members to excel, evolve and go further within their careers.

For more information about the BSDHT, visit www.bsdht.org.uk, call 01788575050 or email enquiries@bsdht.org.uk. 